\title{
Editorial:
}

\section{Bilingual (multilingual) publications and duplicate publications: for or against?*}

\author{
Yue-hong (Helen) ZHANG \\ (Journals of Zhejiang University-SCIENCE (A/B/C), Hangzhou 310027, \\ China) \\ E-mail: jzus@zju.edu.cn
}

doi: $10.1631 /$ jzus.A1300272

From the professional perspective of journal publishing, whether a bilingual (multilingual) publication is suspicious from an ethical point of view has been a topic of debate. Indeed this problem has been long puzzling authors who are preparing for submission. Although the worldwide publishing industry has had some discussions about it, so far in China no normative text or guide is available for authors and journal editors as reference. In fact, it is not easy to sum up the rights and wrongs of duplicate publication and bilingual (multilingual) publication. From a professional perspective, a few highlights for discussion on the criteria for bilingual (multilingual) publication and substantial duplicate publication are proposed and could be a reference for authors and editors.

\section{Rationality and transparency of bilingual (multilingual) publication}

The foreign language versions of the Bible, well-known as the world's best seller, had amounted to 2197 by the year 1997, which at least demonstrates that in the world there were more than 2000 written languages, based on data on www.baike.com. Similarly, among the published books in the world's commonly used languages, there must be translations of Einstein's "Theory of Relativity", showing that

\footnotetext{
* Project supported by the Committee on Publication Ethics (COPE) (December, 2010) and the National Natural Science Foundation of China (No. 30824802)

(C) Zhejiang University and Springer-Verlag Berlin Heidelberg 2013
}

world culture and scientific communication depend on translation and publication in different languages, and thus provide the rationality for multilingual publication.

However, just like other activities of human beings, publishing activity involves ethics and morality problems. Since copyright laws were introduced (Williams, 2007), they have helped formulate the norms of publishing activity. Recently, one article (Zhang Q., 2013) from "Science and Technology Review" elaborated that in the process of Chinese and English bilingual submissions, authors should have credibility, i.e., non-infringement, and manuscripts should be easy to propagate. As a journal editor, combining the practice of publishing and the idea of publishing internationally, I would like to highlight the following points as references for journal editors handling and authors preparing for bilingual submissions, and a report on this topic, in Chinese, was published in Science and Technology Review (Zhang Y.H., 2013).

1. Transparency and permission

Bilingual (multilingual) publication should include the following processes. After publishing one paper in a primary language, authors should get permission from the primary journal (some international journals have already provided an authorization statement in the text), and should state acknowledgement of a secondary publication or which parts are a secondary publication in the manuscript when submitting the manuscript in another language. Such action is regarded to be free of copyright suspicions, faithful to the primary version, and free of any academic misconduct. Then reviewers and journal editors will judge and decide whether the content of this manuscript is useful and necessary as a secondary publication or not.

\section{Achievement and evaluation}

After bilingual (multilingual) publishing, primary and secondary publications should be counted 
only once by relevant research or personnel departments in quantitative assessment of original research achievements by the identified author. This is because the primary publication shows the original research, while the secondary publication is just a translation of it. Of course translations also belong to creative works of language. For example, the translations of famous Chinese translators Mr. FU Lei and Mr. LIN Yu-tang have been of long-term cultural benefit to the readers. Therefore, the secondary publication may be a reference for language skills when relevant departments are assessing achievements.

\section{Indexing and referencing}

Indexing and referencing of primary and secondary publications are to be discussed, respectively. Indexing refers to databases. For the National Library of Medicine (NLM), when the primary and secondary publications both appear in the same journal, editors of journals should understand that MEDLINE/ PubMed indexes the primary language version. For example, some Canadian journals publish manuscripts in both English and French simultaneously, the MEDLINE/PubMed database only indexes abstracts published in English, not in French, but both languages are indicated in the MEDLINE citation (such as, Mercer K. The relentless challenge in health care. Healthcare Management Forum. 2008 Summer, 21(2):4-5. English, French) (ICMJE, 2013). An index of the primal and secondary publications of other journals in different languages may vary, so duplicate abstracts might appear in the same database (Errami and Garmer, 2008). Reference of primary and secondary publications is usually based on the language preference of readers, so generally no special provisions exist.

\section{Discussion of duplicate publications and bilingual (multilingual) publications}

As specified above, although bilingual (multilingual) publication is theoretically reasonable, it will undoubtedly have to be defined as a duplicate publication which is not to be commended if the publishing processes are not transparent. Duplicate publications lead to double-counting in the assessment of research achievements, and moreover lead to fragmentation and misinterpretation of effective academic research, so it is contrary to ethical standards. What are the types of duplicate publication? What are the manifestations of such misconduct? In particular, under what circumstances is multilingual publication wrong? How to conduct this correctly? In one world, one principle of publishing, shall we learn from Table 1, named "Guide to duplicate submission/publication and how to prevent it" from Elsevier (2012) to discuss the rights and wrongs of duplicate publication and multilingual publication.

Analysis and correction of four types of duplicate publication behaviour clearly show that ethical standards of the international academic publishing industry are consistent. In particular, analysis of multilingual publications shows that without preconditions of transparency, multilingual publications might be suspected of infringement and misconduct, and then classified as duplicate publications.

Furthermore, I would like to communicate with my peers about our recent completed research findings from the Committee on Publication Ethics (COPE) Fund (COPE Research Granted, 2011; Zhang and Jia, 2012; 2013; Jia et al., 2013; Zhang Y.H. et al., 2013; Zhang X.X. et al., 2013). In particular, one investigation is that whether manuscripts which have been already published at computing/electronics conference proceedings, then submitted to journals, are duplicate submissions/publications? (Zhang and Jia, 2013). It was the consensus through the 1980s and 1990s in the field of computing and electronics that publishing of conference proceedings, prior to journal publication, contributed to rapid exchange of information, so resubmissions to journals were considered reasonable. However, with the rapid development of the Internet, concepts and techniques of the publishing industry have been updated, and the standards of ethics have been raised, so the norms of resubmissions have become suspect and there has been an attack on duplicate publication in recent years. Prof. Moshe Y. VARDI, the chief editor of the journal Communications of the $A C M$ spoke out on this issue in the editors' forum: "we are driving on the wrong side of the publication road" (Vardi, 2009). Rome was not built in one day. Of course, changes in the norms for resubmissions need time, and consensus about stronger publishing ethics needs to be established and expanded.

Also, one typical case from COPE refers to a discussion of the rights and wrongs of duplicate 
Table 1 Guide to duplicate submission/publication and how to prevent it (Elsevier, 2012) ${ }^{\text {*\# }}$

\begin{tabular}{llll}
\hline \multicolumn{1}{c}{ Action } & \multicolumn{1}{c}{ What it is } & \multicolumn{1}{c}{ Is it unethical? } & \multicolumn{1}{c}{ What should you do? } \\
\hline $\begin{array}{l}\text { Simultaneous } \\
\text { submission }\end{array}$ & $\begin{array}{l}\text { Submitting a paper to } \\
\text { two or more journals at } \\
\text { the same time }\end{array}$ & $\begin{array}{l}\text { Yes. } \\
\text { Submission is not permit- } \\
\text { ted as long as a manuscript } \\
\text { is under review with an- } \\
\text { other journal }\end{array}$ & $\begin{array}{l}\text { 1. Avoid submitting a paper to more than one } \\
\text { publication at a time }\end{array}$ \\
$\begin{array}{lll}\text { 2. Even if a submitted paper is currently } \\
\text { under review and you do not know the } \\
\text { status, wait to hear back from the publisher } \\
\text { before approaching another journal, and } \\
\text { then only if the first publisher will not be } \\
\text { publishing the paper }\end{array}$
\end{tabular}

\begin{tabular}{ll}
\hline Duplicate & When an author submits \\
publication & Yes. \\
& a paper or portions of his \\
& or her own paper that has \\
& been previously pub- \\
& lished to another journal, \\
& without disclosing prior \\
& submission(s)
\end{tabular}

1. Avoid submitting a previously published paper for consideration in another journal

2. Avoid submitting papers that describe essentially the same research to more than one journal

3. Always provide full disclosure about any previous submissions (including conferences, presentations and posting of results in registries) that might be regarded as duplicate publication

4. This should include disclosing previous publication of an abstract during the proceedings of conferences

\begin{tabular}{|c|c|c|c|}
\hline $\begin{array}{l}\text { Duplication by } \\
\text { paraphrasing } \\
\text { or "text- } \\
\text { recycling" }\end{array}$ & $\begin{array}{l}\text { When an author writes } \\
\text { about his or her own } \\
\text { research in two or more } \\
\text { articles from different } \\
\text { angles or on different } \\
\text { aspects of the research } \\
\text { without acknowledgment } \\
\text { of the original paper }\end{array}$ & $\begin{array}{l}\text { Yes. } \\
\text { Creating several publica- } \\
\text { tions from the same re- } \\
\text { search, is considered ma- } \\
\text { nipulative. See our separate } \\
\text { factsheet on plagiarism/text } \\
\text { recycling }\end{array}$ & $\begin{array}{l}\text { 1. Put anything in quotes that is taken di- } \\
\text { rectly from a previously published paper, } \\
\text { even if you are reusing something in your } \\
\text { own words } \\
\text { 2. Make sure to reference the source ac- } \\
\text { cordingly }\end{array}$ \\
\hline $\begin{array}{l}\text { Translations of } \\
\text { a paper pub- } \\
\text { lished in an- } \\
\text { other language }\end{array}$ & $\begin{array}{l}\text { Submitting a paper to } \\
\text { journals in different } \\
\text { languages without ac- } \\
\text { knowledgment of the } \\
\text { original paper }\end{array}$ & $\begin{array}{l}\text { Yes. } \\
\text { Translated articles are ac- } \\
\text { ceptable when all necessary } \\
\text { consents have been ob- } \\
\text { tained from the previous } \\
\text { publisher of the paper in any } \\
\text { other language and from } \\
\text { any other person who might } \\
\text { own rights in the paper }\end{array}$ & $\begin{array}{l}\text { 1. If you want to submit your paper to a } \\
\text { journal that is published in a different } \\
\text { country or a different language, ask the } \\
\text { publisher if this is permissible } \\
\text { 2. At the time of submission, disclose any } \\
\text { details of related papers in a different } \\
\text { language, and any existing translation }\end{array}$ \\
\hline
\end{tabular}

\footnotetext{
${ }^{*}$ When in doubt, always consult with your professor, advisor, or someone in a position of authority who can guide you to the right course of action

${ }^{\text {\# }}$ Reprinted with the permission of the Global Rights Department of Elsevier
}

publications (COPE, 2011). This case involves four manuscripts in which three manuscripts were originally published in authors' native language and then the fourth was published in an English language journal. There was overlap in the authors who were involved in all four manuscripts and who were reported and suspected of duplicate publication. So the English language journal took the lead to investigate. Editors from different language journals collaborated with each other and contacted the authors to investigate and collect evidence, and finally decided to keep the third manuscript available and retract the other three papers, including the English one, due to duplication.

Furthermore, this case demonstrates that in the global village, the ethical standards of academia and the publishing industry are consistent. From another perspective, it also shows that nowadays journal editors should spend quite a lot of energy dealing with such problems, besides normal editing and publishing work. So attaching importance to academic ethics is the common responsibility of the scientific community and publishing industry. 


\section{References}

COPE, 2011. Duplicate Publication in Possibly Four Papers. Case No. 11-21. Available from http://publicationethics. org/case/duplicate-publication-possibly-four-papers [Accessed on Apr. 20, 2013].

COPE Research Granted, 2011. CrossCheck Guidance: An Analysis of Typical Cases of Plagiarism in Different Disciplines. Available from http://publicationethics.org/ resources/research [Accessed on Jan. 26, 2011].

Elsevier, 2012. Simultaneous Submission/Multiple, Duplicate Publication via Ethics in Research and Publication Available from http://www.ethics.elsevier.com/pdf/ ETHICS_SSUB01a.pdf [Accessed on Apr. 15, 2013].

Errami, M., Garmer, H., 2008. A tale of two citations. Nature, 451(7177):397-399. [doi:10.1038/451397a]

ICMJE, 2013. Uniform Requirements for Manuscripts Submitted to Biomedical Journals: Overlapping Publications. Available from http://www.icmje.org/publishing_4overlap. html [Accessed on July, 2013].

Jia, X.Y., Tan, X.F., Zhang, Y.H., 2013. Replication of the methods section in biosciences papers: is it plagiarism? Scientometrics, in press. [doi:10.1007/s11192-013-1033-5]

Vardi, M.Y., 2009. Conferences vs. journals in computing research. Communications of the ACM, 52(5):5. [doi:10. 1145/1506409.1506410]
Williams, A., 2007. Copyright: past, present and future. Learned Publishing, 20(2):113-119. [doi:10.1087/ 174148507X185072]

Zhang, Q., 2013. Reflection on moral issue of submitting the same writing for many publications in different languages. Science \& Technology Review, 31(4):15-16 (in Chinese).

Zhang, X.X., Huo, Z.L., Zhang, Y.H., 2013. Detecting and (not) dealing with plagiarism in an engineering paper: beyond CrossCheck-a case study. Science Engineering Ethics, in press. [doi:10.1007/s11948-013-9460-5]

Zhang, Y.H., 2013. The rights and wrongs of bilingual publications and duplicate publications. Science and Technology Review, 31(16): 17-18 (in Chinese).

Zhang, Y.H., Jia, X.Y., 2012. A survey on the use of CrossCheck for detecting plagiarism in journal articles. Learned Publishing, 25(4):292-307. [doi:10.1087/ 20120408]

Zhang, Y.H., Jia, X.Y., 2013. Republication of conference papers in journals? Learned Publishing, 26(3):189-196. [doi:10.1087/20130307]

Zhang, Y.H., Jia, X.Y., Lin, H.F., Tan, X.F., 2013. Editorial: Be careful! Avoiding duplication: a case study. Journal of Zhejiang University-SCIENCE B (Biomedicine \& Biotechnology), 14(4):355-358. [doi:10.1631/jzus.B1300078]

\title{
Recommended paper related to this topic
}

\section{Editorial: Be careful! Avoiding duplication: a case study}

Authors: Yue-hong Zhang, Xiao-yan Jia, Han-feng Lin, Xu-fei Tan Journal of Zhejiang University-SCIENCE B (Biomedicine \& Biotechnology), 2013, Vol. 14, No. 4, P.355-358 doi:10.1631/jzus.B1300078

\begin{abstract}
In bioscience papers, besides the other scientific misconduct issues, replication of the method section is a common problem because duplication is always being detected in the section Materials and Methods. We editors often receive comments and queries from authors who think that it is a matter of course to copy their own published materials as opposed to copying those of others. How should editors handle such papers with similar content in the method section and how to guide authors in writing the method section without being accused of plagiarism? What is right? What is wrong? Here we studied an example to explain this problem.
\end{abstract}

\section{Suggestions}

Moreover, we also quote the useful words of a bio-scientist (Dr. McCanless) who suggested as follows:

1. If using kits and supplier is referenced, then this is enough due to most suppliers providing manuals.

2. If using traditional/homemade methods include following statement: "based on methods previously described" and include reference(s) as these procedures have all been documented in early chemistry/biochemistry journals.....this is how we know how to do them.

3. If the procedure is novel or incorporates a novel step to a known method provide details (if the entire procedure is novel then a separate publication based on the method would be justified; if minor alterations are performed then state "based off [on] previously detailed protocols with the exception of..." Provide a reference for basic protocol and detail and innovative changes that were required to complete your experiment).

We think the three suggestions would be very useful for the author(s) reference in the biomedicine and biotechnology areas. 